\title{
A Framework for Strategic Cloud Migration
}

\author{
Monjur Ahmed \\ Centre for Information Technology \\ Waikato Institute of Technology (Wintec) \\ Hamilton \\ New Zealand \\ Monjur.Ahmed@wintec.ac.nz
}

\author{
Navjot Singh \\ Centre for Information Technology \\ Waikato Institute of Technology (Wintec) \\ Hamilton \\ New Zealand \\ navsind9@student.wintec.ac.nz
}

\begin{abstract}
This paper presents a novel framework for organisations to carry out a structured feasibility study on Cloud migration and to decide Cloud Migration Strategy. Following the framework helps an organisation to decide whether Cloud migration is a feasible option for them, and if so, the best strategic approach towards Cloud migration. It is a crucial and sensitive part for any organisation to decide whether they should move to Cloud Computing platform. The decision requires strategic approach with proper feasibility study. Several technological, human, security and financial factors are involved in decision making process to move to the Cloud. The proposed framework helps an organisation to carry out a feasibility study to decide whether to move to the Cloud, and if so, what would be the best approach towards Cloud migration. The proposed framework addresses the factors that an organisation must explore to decide on Cloud migration. Cloud Computing has its own pros and cons. A whimsical decision to move to the Cloud may be disastrous for an organisation. Following the proposed framework will help organisations to carry out a structured and integrated feasibility study deal with the decision on Cloud migration.
\end{abstract}

\section{CCS Concepts}

- Information systems $\rightarrow$ Information Systems Applications $\rightarrow$ Computing Platforms - Security and Privacy $\rightarrow$ Human and societal aspects of security and privacy.

\section{Keywords}

Cloud computing; cloud computing feasibility; cloud feasibility; cloud migration; cloud migration strategy.

\section{INTRODUCTION}

Organisations around the globe are moving towards Cloud Computing. Cloud Computing comes with its own advantages and disadvantages. Security is one of the biggest concerns in Cloud Computing [1]-[4]. Cloud Computing comes with many benefits which made it a popular computing approach. This is evident from the major shift towards Cloud Computing by organisations around the globe [4]. Numerous benefits of Cloud Computing do not make it an ultimate choice regardless, since security and other

\footnotetext{
Permission to make digital or hard copies of all or part of this work for personal or classroom use is granted without fee provided that copies are not made or distributed for profit or commercial advantage and that copies bear this notice and the full citation on the first page. Copyrights for components of this work owned by others than ACM must be honored. Abstracting with credit is permitted. To copy otherwise, or republish, to post on servers or to redistribute to lists, requires prior specific permission and/or a fee. Request permissions from Permissions@acm.org.

ICCAI '19, April 19-22, 2019, Bali, Indonesia

(C) 2019 Association for Computing Machinery.

ACM ISBN 978-1-4503-6106-4/19/04 ...\$15.00

DOI: https://doi.org/10.1145/3330482.3330528
}

concerns come in hand-to-hand with the benefits that Cloud Computing offers. For an organisation, a whimsical decision to move towards Cloud Computing may be disastrous. IT/IS strategy and any other kinds of computing strategy should complement business strategy, not the other way around. For an organisation to migrate to the Cloud, the first point of action is to decide whether such migration is feasible for an organisation. The answer to this may not be a direct and straightforward one. Several factors are involved for an organisation to decide whether moving towards Cloud Computing is feasible and safe. Various decision making involves in the process of a safe migration to the Cloud [5]. A strategic feasibility study on whether to migrate to the Cloud, and if so, how to migrate are the core and initial requirements. For this, organisations need a strategic framework to aid in decision making process for Cloud migration. Such a framework can aid in an informed, integrated and structured decision-making process for Cloud migration.

In this paper, we propose a framework for organisations to carry out a structured feasibility study - to decide on whether migration to Cloud platform is an acceptable option, and if so, what would be the Cloud Migration Strategy. The framework explores the factors that an organisation must address to decide on Cloud migration. Cloud Computing has its own pros and cons. A whimsical decision to move to the Cloud may be disastrous for an organisation. Following the proposed framework will help organisations to carry out a structured and integrated feasibility study to decide on Cloud migration.

The rest of the paper is organised as follows: a literature review is presented in the Related Study section. The proposed framework is illustrated and explained in The Framework section followed by Future Developments section that outlines envisioned further research on the framework.

\section{RELATED STUDY}

According to [6], a quality score must be calculated by considering all the important factors governing the cloud migration. These factors can be technology used, expenses, learning curves etc. They propose a Cloud-RMM Reference Model which will be developed based on prior experience using a bottom up approach. This model will help in identifying the key process involved in the cloud migration of an IT project. They also recommend a characterisation framework for cloud migration to analyse potential approaches that can be used for cloud migration.

As stated in [7], cloud servers provide an unpredictable environment that may not be accessible sometimes due to technical problems. A cloud migration framework must provide appropriate measures to handle these problems. In addition to this, the software developers should program the IT projects to become resilient to such unprecedented situations [8]. 
Data protection is an important aspect to consider in Cloud Computing. The cloud servers keep shifting the deployed projects to different servers while performing load balancing operations [9]. Sometimes, it is difficult to predict from which location the deployed project is running on the cloud server [10]. Therefore, software developers must provide the appropriate measure to protect the sensitive data of users and the enterprise [7].

Different cloud servers use different technology stack and tools for deploying projects to them [7]. Software developers needs to learn new tools while shifting an application from one cloud server to another [11]. Organizations must spend a lot of time and money on this. Therefore, a cloud migration framework will provide a unified way of deploying projects across various cloud servers and make the deployment easy for developers [12].

Legacy applications of enterprise are usually very old projects that uses obsolete technologies. According to [7], the legacy applications are usually incompatible with current technology stack of the cloud servers. Many times, the enterprises must refactor and change the legacy code to work on cloud server, which is a very cumbersome work [13]. Therefore, a cloud migration framework must provide suitable integrators and adaptors that facilitates the migration of legacy application without making big changes in the source code [10].

The existing technical approaches for Cloud migration process found are re-hosting, re-platforming, re-purchasing and refactoring [14]. Re-hosting is moving the software projects of an organization to better hosting server while preserving all the business logic of the software projects [15]. Re-hosting involves the planning of current software projects as per requirements of the new hosting server. Usually some changes are needed to be done in the configurations of projects to integrate them with the target environment. Re-hosting also involves the migration of data used by the projects from existing hosting server to the new hosting server. However, the projects already running in the cloud servers are easy to re-architect and deploy on the new hosting server [16]. According to [16], re-platforming involves making cloud-based optimization to software projects while deploying them on a new platform. Re-platforming on modern cloud servers usually does not need to change the basic architecture of the software project. According to [15], re-platforming is used by organizations to convert and integrate various components and business data of a software project. Usually these projects are deployed on test environments during the transition phase, once testing is done, they are deployed on the production environment. Re-platforming has always helped the organizations to enhance the software projects at lesser costs than re-factoring [17]. Repurchasing is done by organizations to move on a different product and a new license model other than the existing one. This also involvement an upgradation of features and a better software implementation methodology. It must be noted that organizations will have to bear an extra cost for obtaining new licenses for the products and this cost depends upon the product and its license plans. Re-factoring is usually done in cloud migration to enhance the current software project [16]. It involves customizing existing software to comply with the software stack and tools used on the cloud server. It can be an expensive process if large amount of changes is to be made in the software. Re-factoring is always needed when a software project is evolving and trying to support the new requirements and expectations of the users [17].

To the best of the authors' knowledge, a framework for Cloud migration does not exist to date that encapsulates organisational, people, cost, security and technological factors to realise the feasibility of Cloud migration and the suitable strategic approach for such migration. The presented framework fill-in this gap.

\section{THE FRAMEWORK}

The decision to migrate to the Cloud for an organisation needs to be scrutinised based on several factors. These factors relate to an organisation's mission and vision and its existing capability, the technology used, and the costs involved. A justification of these aspects would first give a 'yes' or 'no' answer to the question: 'Should we migrate to Cloud Computing?'

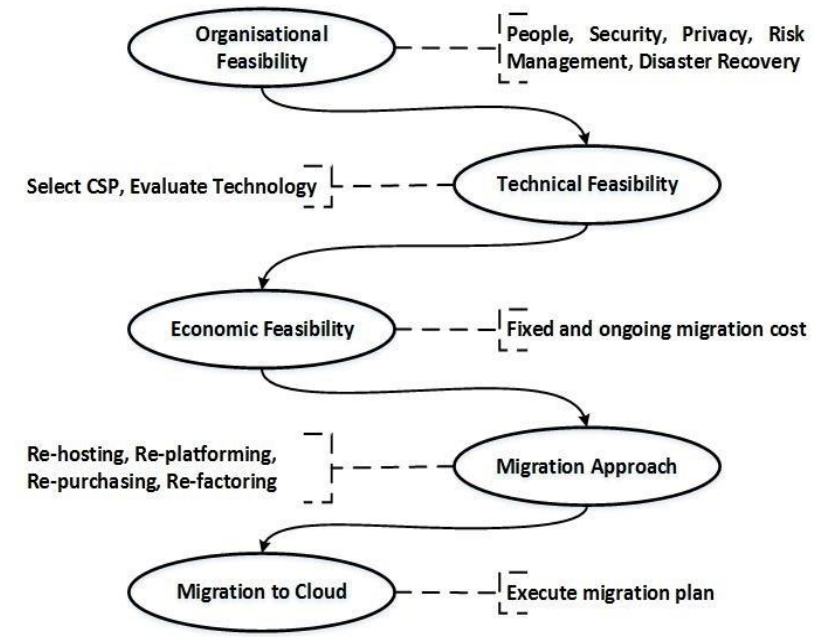

Figure 1. Strategic framework for cloud migration.

Figure 1 shows the proposed strategic cloud migration framework. The major stages in the framework are Organisational feasibility, Technical feasibility, Economic Feasibility, Migration Approach and Migration to Cloud. Organisational, Technical and Economic Feasibility leads for an organisation to realise whether they should move to the Cloud, and if so, the best suited approach is explored through Migration Approach after which the actual migration to the Cloud takes place. If Organisational Feasibility yields negative outcome (i.e. a 'no' answer towards Cloud migration), an organisation does not require to proceed further towards exploring whether they should migrate to the Cloud. Otherwise, the phase Technical Feasibility takes place. Again, if the outcome of Technical Feasibility phase is negative, an organisation should stop proceeding further and scrap the decision to migrate to the Cloud. An organisation may proceed further towards Cloud migration if and only if the outcome of the feasibility for both Organisational feasibility and Technical Feasibility indicates assertiveness. Unlike the first two phases mentioned above, Economic Feasibility analysis is rather a trade-off analysis and subjective decision point for an organisation. Incurring more costs comparing to non-Cloud architecture may not be a sole reason for an organisation to refrain from Cloud migration. On completion of this phase, if an organisation finds it viable to move to the Cloud, the approaches through which the actual migration will take place is considered and an organisation starts the process to migrate to the Cloud.

To carry out Organisational Feasibility to decide worthiness or Cloud migration, an organisation needs to consider five factors: People, Security, Privacy, Risk management, and Disaster Recovery. It is important to evaluate the employees' current skillset and whether there will be any skill gap due to Cloud migration. If any skill gap identified, whether they will be fulfilled through training or through hiring new employees are factors to be 
considered. This will further be linked into the fixed migration cost discussed later.

Security and privacy are perhaps the most important and most notorious and prioritised factors to explore. These two factors, if not judged from a feasibility point-of-view, may result for an organisation in losing everything in the long or short run. If migrating to Cloud Computing means shifting all digital assets to the Cloud, and since moving to the Cloud (except private Cloud) essentially means putting all resources into someone else's computer under someone else's management, an organisation must not leave even a tiny fraction of rom to compromise on security and privacy on deciding to migrate to the Cloud. To mention a few, the reputation of the chosen CSP, the location of the data centre (not CSP's office, the actual data centre) and which region's legislation applies to the data centre, what are the policies and regulations of the CSP, and to what extent a CSP is accountable by the local laws in the region they are located in these are factors to be considered when it comes to security and privacy, as well as to choose a CSP to partner with if the decision to migrate to the Cloud is a 'yes'. Thus, exploring the impact of security and privacy is also linked to selecting a CSP that is part of Technical Feasibility phase of the proposed framework, as illustrated in Figure 1.

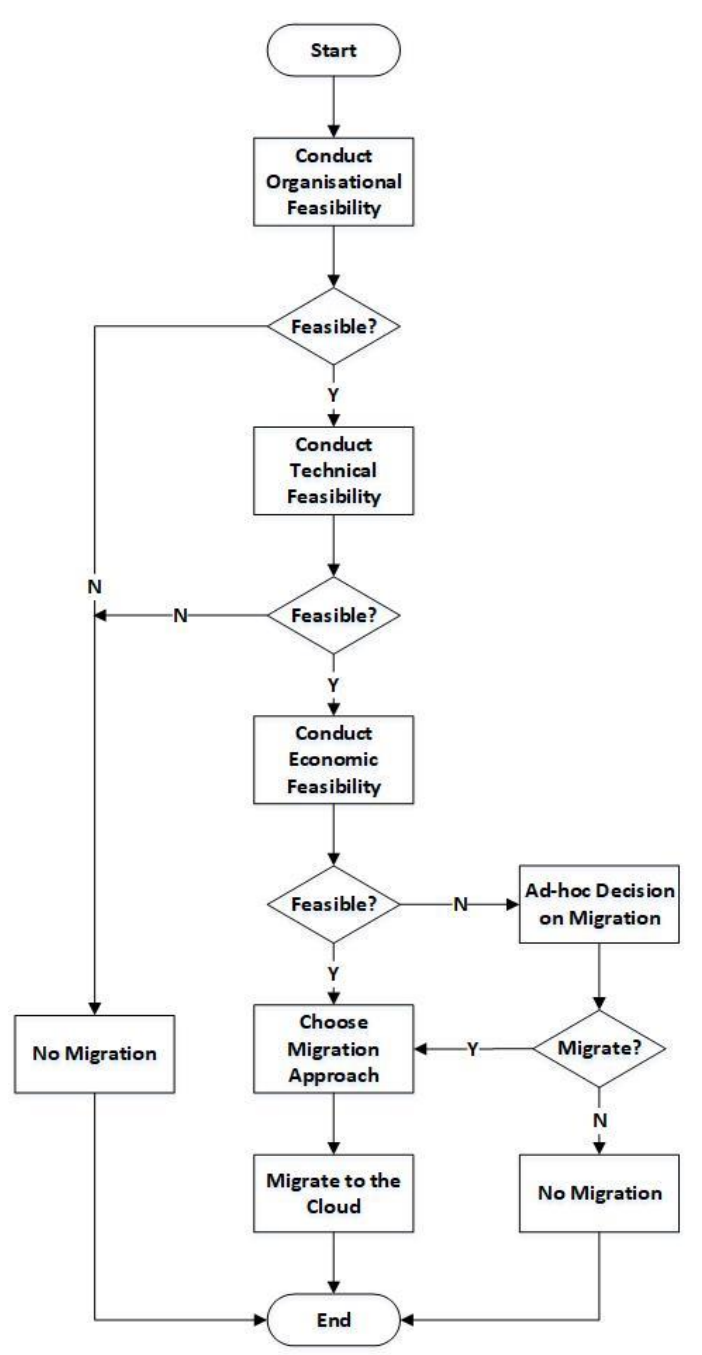

Figure 2. Decision making flow for cloud migration.
Migrating to the Cloud involves moving most (if not all) of an organisation's digital assets to CSP's infrastructure. It is crucial to realise what such migration would mean for the organisation's current risk management and disaster recovery procedures, and whether there will be changes introduced to these procedures. If changes are introduced, what impact would the changes have in terms of integrity of the existing risk management and disaster recovery procedures? An organisation needs to find solid answers to these questions to determine feasibility to migrate to the Cloud.

Table 1. Key questions for cloud migration planning

\begin{tabular}{|c|c|}
\hline \multicolumn{2}{|r|}{ Organisational Feasibility } \\
\hline People & $\begin{array}{l}\text { Are our people skilled to operate as normal } \\
\text { once we moved to the Cloud? What would } \\
\text { be the skills gaps after we move to the } \\
\text { Cloud? }\end{array}$ \\
\hline Security & $\begin{array}{l}\text { How moving to the Cloud would impact on } \\
\text { the security of our digital assets that we will } \\
\text { store on the Cloud? Will we have to partner } \\
\text { with other third-party organisations apart } \\
\text { from the CSP? How this partnership would } \\
\text { impact the security of our digital assets? }\end{array}$ \\
\hline Privacy & $\begin{array}{l}\text { Will we have more integrated and better } \\
\text { digital privacy if we move to the Cloud? }\end{array}$ \\
\hline $\begin{array}{l}\text { Risk } \\
\text { Management }\end{array}$ & $\begin{array}{l}\text { Will existing risk management procedures } \\
\text { have to be changed if we move to the } \\
\text { Cloud? If so, will that be more robust and } \\
\text { are we capable of managing new Risk } \\
\text { Management procedures once we move to } \\
\text { the Cloud? }\end{array}$ \\
\hline $\begin{array}{l}\text { Disaster } \\
\text { Recovery }\end{array}$ & $\begin{array}{l}\text { Will current disaster recovery procedures } \\
\text { change as a result of moving to the Cloud? } \\
\text { Are we able to manage the disaster recovery } \\
\text { procedures if we move to the Cloud? }\end{array}$ \\
\hline \multicolumn{2}{|r|}{ Technical feasibility } \\
\hline Select CSP & $\begin{array}{l}\text { Based on which factors we are selecting this } \\
\text { CSP? How these factors to our } \\
\text { organisation's mission and vision? }\end{array}$ \\
\hline $\begin{array}{l}\text { Evaluate } \\
\text { Technology }\end{array}$ & $\begin{array}{l}\text { What technologies are used by our chosen } \\
\text { CSP? How they are similar and different } \\
\text { compared to their (i.e. CSP) competitors? } \\
\text { What are the weaknesses/vulnerabilities } \\
\text { inherent in these technologies? Will these } \\
\text { technologies pose any security threat to } \\
\text { digital assets? }\end{array}$ \\
\hline \multicolumn{2}{|r|}{ Economic Feasibility } \\
\hline $\begin{array}{l}\text { Fixed } \\
\text { Migration Cost }\end{array}$ & How much is the fixed migration cost? \\
\hline Ongoing Cost & $\begin{array}{l}\text { How much will be the ongoing cost? Given } \\
\text { all other aspects and their } \\
\text { benefits/drawbacks, and considering fixed } \\
\text { migration cost and ongoing cost, does it } \\
\text { make sense to migrate to the Cloud, and if } \\
\text { so, how it does so? }\end{array}$ \\
\hline \multicolumn{2}{|r|}{ Migration to the Cloud } \\
\hline Approach & Which approach is best suited and why? \\
\hline
\end{tabular}

The Select CSP step in Technical Feasibility phase is discussed earlier. In addition to that, an evaluation of the technologies used by the CSP also demands scrutinising. Vulnerabilities in soft and hard tools used for a Cloud infrastructure may pose threats to digital assets housed in a Cloud infrastructure. The technologies 
related to software, operating systems, network protocol, firmware, firewall and everything must be scrutinised to ascertain whether they may pose any security threat to digital assets due to their inherent weakness or vulnerabilities. As part of Economic Feasibility, an analysis on fixed and ongoing costs as a result of Cloud migration is figured out to further decide whether to continue with Cloud Migration planning or not.

If the case passes all the aforementioned phases, the Migration Approach can be selected. To the best of the authors' knowledge, there is no specific rule of thumb or a standard on Cloud migration approaches. However, the options to choose from that are gaining familiarity are re-hosting, re-platforming, repurchasing and re-factoring. These are discussed earlier in Related Study section. Figure 2 illustrates the decision-making process for Cloud migration using the framework.

As part of the migration process, an organization needs to ask questions for all the factors related to Cloud migration to work out the feasibility of the migration. A non-exhaustive list of such questions is illustrated in Table 1.

Answering the questions listed in Table 1 helps an organisation to realise the impact of Cloud migration for them. The list of questions in table 1 are indicative only, and an organisation is at their freedom to add questions that would help them to satisfactorily reach a decision on whether to migrate to the Cloud or not.

\section{FUTURE DEVELOPMENTS}

As part of future development, we aim to formulate a quantitative model with KPIs to calculate predictions on different phases of cloud migration strategy planning. A case study for Cloud Migration will be considered to demonstrate to ascertain the level of integrity inherent in the developed quantitative model. Part of the future development will involve exploring the option to build industry-specific (e.g. education, healthcare, banking) blueprint of the model that organisations can use with no or little modifications.

\section{CONCLUSIONS}

The presented framework structures various factors as well as Cloud migration approaches and combine them into a common framework for a seamless strategic decision towards Cloud migration - this is where the novelty of the proposed framework stands. The framework helps an organisation to take informed and integrated decision towards Cloud migration. The decision to migrate to the Cloud is a strategic approach rather than a mere technological move. Where technical aspects, of course, play an important role in performance and operational efficiency, they are of simply no value if strategic aspects (e.g. security, risk management) are planned in an integrated manner. When it comes to migrating to the Cloud, for an organisation the decision might very well be a resounding 'no'. A whimsical move towards Cloud migration without strategic analysis may lead to irrecoverable disaster for an organisation, and this can happen by accepting any level of compromise to security and privacy during the Cloud migration process. On Cloud migration, the process of decision making by following a framework may be more effort demanding than the actual move to the Cloud itself, but this is something an organisation must not compromise with.

\section{REFERENCES}

[1] Zissis, D., \& Lekkas, D. 2012. Addressing cloud computing security issues. Future Generation Comp. Syst., 28, 583-592.
[2] Chen, Y., Paxson, V., \& Katz, R.H. 2010. What's New About Cloud Computing Security? Technical Report. Electrical Engineering and Computer Sciences, University of California at Berkeley.

[3] Sabahi, F. 2011. Cloud computing security threats and responses. 2011 IEEE 3rd International Conference on Communication Software and Networks, 245-249.

[4] Ali, M., Khan, S.U., \& Vasilakos, A.V. 2015. Security in cloud computing: Opportunities and challenges. Inf. Sci., 305, 357-383.

[5] Cardoso, A., Moreira, F., \& Escudero, D.F. 2017. Information Technology Infrastructure Library and the migration to cloud computing. Universal Access in the Information Society, 17, 503-515.

[6] Jamshidi, P., Ahmad, A., \& Pahl, C. 2014. Cloud Migration Research: A Systematic Review. Transactions on Cloud Computing. doi:10.1109/TCC.2013.10

[7] Gholami, M. F., Daneshgar, F., Low, G., \& Beydoun, G. (2016, October). Cloud migration process - A survey, evaluation framework, and open. The Journal of Systems \& Software. 31-69. doi:10.1016/j.jss.2016.06.068

[8] García-Galán, J., Trinidad, P., Rana, O. F., \& Ruiz-Cortés, A. 2016. Automated configuration support for infrastructure migration to the cloud. Future Generation Computer Systems. 200-212. doi:10.1016/j.future.2015.03.006

[9] Khan, N., \& Al-Yasiri, A. 2016. Framework for cloud computing adoption: A road map for SMEs to cloud migration. 5. doi:10.5121/ijccsa.2015.5601

[10] Bazi, H. R., Hassanzadeh, A., \& Moeini, A. 2017. A comprehensive framework for cloud computing migration using Meta-synthesis approach. 87-105. doi:10.1016/j.jss.2017.02.049

[11] Hababeh, I. 2015. Data Migration among Different Clouds. Retrieved from http://arxiv.org/abs/1512.08383

[12] Glasgow, P. (2017). De-risking cloud migration. Data Processing, Hosting, and Related Services, 8-8.

[13] Alruwaili, F. F., \& Gulliver, T. A. 2018. Secure migration to compliant cloud services: A case study. Journal of Information Security and Applications. 50-64. doi:10.1016/j.jisa.2017.11.004

[14] Suleman, A. 2018. The Best Cloud Migration Path: Lift And Shift, Replatform Or Refactor? Forbes Technology Council. Available at: https://www.forbes.com/sites/forbestechcouncil/2018/03/23/t he-best-cloud-migration-path-lift-and-shift-replatform-orrefactor/\#38fce7ec4f51. Accessed on 30/October/2018

[15] Oracle. 2018. Oracle Tuxedo Application Rehosting Workbench. Retrieved from: https://docs.oracle.com/cd/E18050_01/artwb/docs11gr1/proc ess/Introduction.html. Accessed on 29 November 2018.

[16] AWS. 2018. Migrating to Amazon Web Services. Retrieved from AWS: https://aws.amazon.com/cloud-migration/. Accessed on 25 November 2018

[17] Bavare, A. 2017. 3 strategies for migrating applications to the cloud. Accenture. Retrieved from Accenture: https://www.accenture.com/us-en/blogs/blogs-amod-bavarekeys-to-refactoring-cloud-migration. Accessed on 25 November 2018. 